TRANSACTIONS OF THE

AMERICAN MATHEMATICAL SOCIETY

Volume 362, Number 1, January 2010, Pages 477-490

S 0002-9947(09)04816-8

Article electronically published on August 12, 2009

\title{
PROJECTIVE NORMALITY OF THE MODULI SPACE OF RANK 2 VECTOR BUNDLES ON A GENERIC CURVE
}

\author{
TAKESHI ABE
}

\begin{abstract}
We prove that the embedding to the projective space by the generalized theta divisors of the moduli space of rank 2 vector bundles on a generic curve is projective normal.
\end{abstract}

\section{INTRODUCTION}

Let $C$ be a smooth projective irreducible curve of genus $g \geq 2$ over an algebraically closed field $k$ of characteristic zero. Let $S U_{C}(2)$ be the moduli space of semistable vector bundles of rank 2 with trivial determinant on $C$. Then we have $\operatorname{Pic} S U_{C}(2) \simeq \mathbb{Z}[\mathcal{L}]$ with $\mathcal{L}$ the ample generator $(\overline{\mathrm{D}-\mathrm{N}})$. Global sections of $\mathcal{L}$ are called generalized theta functions as an analogue to ordinary theta functions on the Jacobian of $C$.

By $\underline{\mathbb{R}}$, the complete linear system $|\mathcal{L}|$ is base-point free. So we have a morphism

$$
\varphi_{\mathcal{L}}: S U_{C}(2) \rightarrow \mathbb{P}\left(\mathrm{H}^{0}\left(S U_{C}(2), \mathcal{L}\right)\right) .
$$

It is known that

- if $g=2$, then $\varphi_{\mathcal{L}}$ is an isomorphism ( $\left.[\mathrm{N}-\mathrm{R}]\right)$,

- if $g \geq 3$ and $C$ is hyperelliptic, then $\varphi_{\mathcal{L}}$ is not an embedding (B1]),

- if $g \geq 3$ and $C$ is not hyperelliptic, then $\varphi_{\mathcal{L}}$ is an embedding ( $[\mathrm{VG}-\mathrm{I}],[\mathrm{B}-\mathrm{V}]$ ).

In this paper we consider the projective normality of $\varphi_{\mathcal{L}}$, that is, the surjectivity of the multiplication map

$$
\mu_{n}: S^{n} \mathrm{H}^{0}\left(S U_{C}(2), \mathcal{L}\right) \rightarrow \mathrm{H}^{0}\left(S U_{C}(2), \mathcal{L}^{\otimes n}\right) .
$$

As to the map $\mu_{n}$, it is known that

- if $C$ has no vanishing thetanull, then $\mu_{2}$ is an isomorphism ([B2] $)$,

- if $C$ has no vanishing thetanull, then $\mu_{4}$ is surjective ( $\left.\mathrm{vG}-\mathrm{P}\right)$,

- if $C$ is generic, then $\mu_{n}$ is surjective for $n$ even and $n \geq 2 g-4$ ([L).

The main result (Theorem 4.2) of this paper is that if $C$ is generic, then the multiplication maps

$$
S^{l} \mathrm{H}^{0}\left(S U_{C}(2), \mathcal{L}^{2}\right) \rightarrow \mathrm{H}^{0}\left(S U_{C}(2), \mathcal{L}^{\otimes 2 l}\right)
$$

Received by the editors November 2, 2007 and, in revised form, May 2, 2008.

2000 Mathematics Subject Classification. Primary 14H60, 14D20.

The author was partially supported by the Japanese Ministry of Education and Science, Grantin-Aid for Young Scientists (B).

(C)2009 American Mathematical Society Reverts to public domain 28 years from publication 
and

$$
\mathrm{H}^{0}\left(S U_{C}(2), \mathcal{L}\right) \otimes S^{l} \mathrm{H}^{0}\left(S U_{C}(2), \mathcal{L}^{2}\right) \rightarrow \mathrm{H}^{0}\left(S U_{C}(2), \mathcal{L}^{\otimes 2 l+1}\right)
$$

are surjective for all $l \geq 1$. Combining this with Beauville's surjectivity of $\mu_{2}$ for curves with no vanishing thetanull ([B2] $)$, we have

Theorem 1.1. $\varphi_{\mathcal{L}}$ is a projectively normal embedding for a generic curve.

1.1. Factorization theorems. Theorems that express the space of generalized theta functions on the moduli space of bundles on a nodal curve in terms of generalized theta functions on the moduli space of parabolic bundles on the normalized curve are called factorization theorems. Factorization theorems for $G L(r)$-bundles have been obtained by Narasimhan-Ramadas ( $[\mathrm{N}-\mathrm{Rd}]$ ) and Sun ([S1], [S2]). A factorization theorem for vector bundles of rank 2 with fixed determinant played an important role in the proof of $S L(2)-G L(n)$ strange duality in $\mathrm{A}$ (see Bel] and M-O for other elegant proofs of strange duality), and it also plays a key role in this paper.

Let $C^{\prime}:=C_{1} \cup C_{2}$ be a nodal curve such that $C_{1}$ and $C_{2}$ are smooth projective curves and they intersect at only one point $p$. Let $L$ be a line bundle on $C^{\prime}$, and put $L_{i}:=\left.L\right|_{C_{i}}$. Let $\mathcal{S U}\left(C^{\prime}, L\right)$ be the moduli stack parametrizing torsion-free sheaves $E$ on $C^{\prime}$ with skew-symmetric isomorphism $E \rightarrow \mathcal{H o m}(E, L)$. We denote by $\Xi_{C^{\prime}}$ the determinant line bundle on $\mathcal{S U}\left(C^{\prime}, L\right)$.

The factorization theorem that concerns us in this paper claims the existence of a filtration

$$
\mathrm{H}^{0}\left(\mathcal{S U}\left(C^{\prime}, L\right), \Xi_{C^{\prime}}^{\otimes n}\right)=V_{n} \supset V_{n-1} \supset \cdots \supset V_{0} \supset V_{-1}=\{0\}
$$

such that for each graded part we have a canonical isomorphism

$$
V_{a} / V_{a-1} \simeq \mathrm{H}^{0}\left(\mathcal{P S U}\left(C_{1}, L_{1} ; p\right), \Xi_{C_{1}, a}^{(n)}\right) \otimes \mathrm{H}^{0}\left(\mathcal{P} \mathcal{S U}\left(C_{2}, L_{2} ; p\right), \Xi_{C_{2}, a}^{(n)}\right) .
$$

Here $\mathcal{P S U}\left(C_{i}, L_{i} ; p\right)$ is the moduli stack of parabolic rank 2 bundles with determinant $L_{i}$ on $C_{i}$, and $\Xi_{C_{i}, a}^{(n)}$ is a certain line bundlle on $\mathcal{P} \mathcal{S U}\left(C_{i}, L_{i} ; p\right)$ (see Definition 2.1).

1.2. Outline of the proof of the main result. In this subsection we shall give an outline of the proof of the surjectivity of the morphisms (1.3) and (1.4).

The strategy of proof is using a degeneration argument and induction on the genus. Let $C^{\prime}$ and $L$ be as in the previous subsection. Consider the multiplication map

$$
S^{n} \mathrm{H}^{0}\left(\mathcal{S U}\left(C^{\prime}, L\right), \Xi_{C^{\prime}}\right) \rightarrow \mathrm{H}^{0}\left(\mathcal{S U}\left(C^{\prime}, L\right), \Xi_{C^{\prime}}^{\otimes n}\right) .
$$

The target has the filtration (1.5), and we let

$$
\mathrm{H}^{0}\left(\mathcal{S U}\left(C^{\prime}, L\right), \Xi_{C^{\prime}}\right)=W_{1} \supset W_{0} \supset W_{-1}=\{0\}
$$

be the filtration for $n=1$.

As will be stated in Proposition 3.1 the filtrations have certain compatibility with respect to the multiplication map. Hence for the surjectivity of (1.7), we have only to consider the surjectivity of the graded parts:

$$
S^{a}\left(W_{1} / W_{0}\right) \otimes S^{n-a}\left(W_{0} / W_{-1}\right) \rightarrow V_{a} / V_{a-1} .
$$


By the isomorphism (1.6), this morphism can be written as

$$
\begin{aligned}
& S^{a}\left(\mathrm{H}^{0}\left(\mathcal{P} \mathcal{S U}_{1}, \Xi_{1}^{(1)}\right) \otimes \mathrm{H}^{0}\left(\mathcal{P} \mathcal{S U}_{2}, \Xi_{1}^{(1)}\right)\right) \quad \mathrm{H}^{0}\left(\mathcal{P} \mathcal{S U}_{1}, \Xi_{a}^{(n)}\right) \\
& \otimes \quad \rightarrow \quad \otimes \\
& S^{n-a}\left(\mathrm{H}^{0}\left(\mathcal{P} \mathcal{S U}_{1}, \Xi_{0}^{(1)}\right) \otimes \mathrm{H}^{0}\left(\mathcal{P} \mathcal{S U}_{2}, \Xi_{0}^{(1)}\right)\right) \quad \mathrm{H}^{0}\left(\mathcal{P} \mathcal{S U}_{2}, \Xi_{a}^{(n)}\right)
\end{aligned}
$$

where we wrote $\mathcal{P S \mathcal { U } _ { i }}$ for $\mathcal{P} \mathcal{S U}\left(C_{i}, L_{i} ; p\right)$.

This is a (tensor product of) multiplication map of generalized theta functions on the moduli space of (parabolic) bundles on $C_{i}$, so we can apply the induction hypothesis.

This naive idea of proof, however, does not work straightforwardly because $\mathrm{H}^{0}\left(\mathcal{P} \mathcal{S U}_{i}, \Xi_{b}^{(1)}\right)=0$ if $\operatorname{deg} L_{i}+b$ is odd, so the source of the morphism (1.10) is zero for $0<a<n$.

This is why we consider the morphisms (1.3) and (1.4) instead of (1.2). Actually in this paper, we apply the above argument to the morphisms (1.3) and (1.4), and prove the surjectivity.

In order to make the induction work, we need to start not from $C$ but from a pointed curve. As the first step of the induction, we prove the surjectivity of a parabolic version of the morphisms (1.3) and (1.4) for one or two-pointed curves of genus one (Proposition 4.1).

\subsection{This paper is organized as follows.}

In section 2 we define a moduli stack of parabolic 2-bundles with fixed determinant and a line bundle on this moduli stack. For a reductive algebraic group $G$ in general, it is a difficult problem to compactify the moduli of $G$-bundles on a nodal curve. For $G=\mathrm{Sp}_{2 r}$ or $\mathrm{O}_{n}$, Faltings [F] considered the moduli of torsion-free sheaves with a non-degenerate bilinear form. Our definition of the moduli stack of parabolic 2-bundles with fixed determinant is just a modification of Falting's moduli in the case $G=\mathrm{Sp}_{2}=\mathrm{SL}_{2}$.

In section 3 we state the factorization theorem of generalized theta functions on the moduli space of 2-bundles with fixed determinant, which will play a central role in the proof of the main theorem. The factorization theorem was proved implicitly in $\mathrm{A}$, but even the statement of the factorization theorem was not stated explicitly there. (It was buried in the proofs of several propositions.) Although we do not need its proof for reading the later sections, we sketch an outline of it, giving explicit reference to $\mathrm{A}$ ] for some technical points.

In section 4 we prove the main theorem. In Proposition 4.1 we treat multiplication maps in the genus one case. In Theorem 4.2 we prove the surjectivity of the multiplications maps (1.3) and (1.4) by induction on the genus.

\section{Moduli of PARABolic BUndles}

As explained in the introduction, we shall prove the main theorem by a degeneration argument. In the course of the proof, parabolic bundles appear naturally. So we first define a moduli stack of parabolic bundles and the line bundles on it.

Definition 2.1. (1) Let $C$ be a connected projective nodal curve over an algebraically closed field $k$ of characteristic zero, and let $s_{1}, \ldots, s_{m}$ be distinct smooth closed points of $C$. Let $L$ be a line bundle on $C$. The moduli stack 
$\mathcal{P S U}\left(C, L ; s_{1}, \ldots, s_{m}\right)$, or $\mathcal{P} \mathcal{S U}$ for short, of parabolic 2-bundles with fixed determinant $L$ is defined as follows. For an affine scheme $T$, an object of the groupoid $\mathcal{P S U}(T)$ is a triple

$$
\left(\mathcal{E}, \mathcal{E} \otimes \mathcal{E} \rightarrow L_{T},\left\{\left.\mathcal{R}_{i} \subset \mathcal{E}\right|_{s_{i} \times T}\right\}_{i=1}^{m}\right)
$$

where $\mathcal{E}$ is a $T$-flat sheaf on $C \times T$ whose restriction to each geometric fiber over $T$ is a torsion-free sheaf of rank $2, \mathcal{E} \otimes \mathcal{E} \rightarrow L_{T}$ is a non-degenerate alternate bilinear form, and $\left.\mathcal{R}_{i} \subset \mathcal{E}\right|_{s_{i} \times T}$ is a line subbundle. (Here the non-degeneracy of $\mathcal{E} \otimes \mathcal{E} \rightarrow L_{T}$ means, by definition, that the induced morphism $\mathcal{E} \rightarrow \mathcal{H o m}\left(\mathcal{E}, L_{T}\right)$ is an isomorphism.)

(2) For integers $n, a_{1}, \ldots, a_{m}$, we define the line bundle $\Xi_{a_{1}, \ldots, a_{m}}^{(n)}$ on $\mathcal{P S U}\left(C, L ; s_{1}, \ldots, s_{m}\right)$ to be

$$
\left(\operatorname{det} \mathbb{R} p r_{*} \mathcal{E}^{u n i v}\right)^{\otimes(-n)} \otimes \bigotimes_{i=1}^{m}\left(\left.\mathcal{E}^{u n i v}\right|_{s_{i} \times \mathcal{P} \mathcal{S U}} / \mathcal{R}_{i}^{\text {univ }}\right)^{\otimes a_{i}}
$$

where we denoted by $\left(\mathcal{E}^{\text {univ }}, \mathcal{E}^{\text {univ }} \otimes \mathcal{E}^{\text {univ }} \rightarrow L_{\mathcal{P} \mathcal{S} \mathcal{U}},\left\{\left.\mathcal{R}_{i}^{\text {univ }} \subset \mathcal{E}^{\text {univ }}\right|_{s_{i} \times \mathcal{P} \mathcal{S U}}\right\}_{i=1}^{m}\right)$ the universal family over $\mathcal{P S U}$, and $p r: C \times \mathcal{P S U} \rightarrow \mathcal{P S U}$ is the projection.

Remark 2.2. As Faltings noted in $[\mathbf{F}$, for $r \geq 3$, it is difficult to compactify the moduli of $\mathrm{SL}_{r}$-bundles on a nodal curve. For recent advances in compactification of $G$-bundles, see Bho1, Bho2, Sch1, Sch2.

Lemma 2.3. Retain the notation as above.

(1) Let $n, a_{1}, \ldots, a_{m}, n^{\prime}, a_{1}^{\prime}, \ldots, a_{m}^{\prime}$ be integers. We have a natural isomorphism

$$
\Xi_{a_{1}, \ldots, a_{m}}^{(n)} \otimes \Xi_{a_{1}^{\prime}, \ldots, a_{m}^{\prime}}^{\left(n^{\prime}\right)} \simeq \Xi_{a_{1}+a_{1}^{\prime}, \ldots, a_{m}+a_{m}^{\prime}}^{\left(n+n^{\prime}\right)}
$$

of line bundles on $\mathcal{P S U}\left(C, L ; s_{1}, \ldots, s_{m}\right)$.

(2) If $f: \mathcal{P S U}\left(C, L ; s_{1}, \ldots, s_{m}\right) \rightarrow \mathcal{P S U}\left(C, L ; s_{1}, \ldots, s_{m-1}\right)$ is the morphism that associates $\left(E, E \otimes E \rightarrow L,\left\{\left.R_{i} \subset E\right|_{s_{i}}\right\}_{i=1}^{m-1}\right)$ to $\left(E, E \otimes E \rightarrow L,\left\{\left.R_{i} \subset E\right|_{s_{i}}\right\}_{i=1}^{m}\right)$, then we have a natural isomorphism

$$
f^{*} \Xi_{a_{1}, \ldots, a_{m-1}}^{(n)} \simeq \Xi_{a_{1}, \ldots, a_{m-1}, 0}^{(n)}
$$

of line bundles on $\mathcal{P} \mathcal{S U}\left(C, L ; s_{1}, \ldots, s_{m}\right)$, and an isomorphism

$\mathrm{H}^{0}\left(\mathcal{P} \mathcal{S U}\left(C, L ; s_{1}, \ldots, s_{m-1}\right), \Xi_{a_{1}, \ldots, a_{m-1}}^{(n)}\right) \rightarrow \mathrm{H}^{0}\left(\mathcal{P} \mathcal{S U}\left(C, L ; s_{1}, \ldots, s_{m}\right), \Xi_{a_{1}, \ldots, a_{m-1}, 0}^{(n)}\right)$.

Sketch of proof. (1) This is obvious from the definition of the line bundles.

(2) The fact that (2.3) is an isomorphism follows from the fact that $f$ is a $\mathbb{P}^{1}$ bundle.

Here we prove a lemma about elementary transformations that will be used in the proof of Proposition 4.1.

Lemma 2.4. Retain the notation in Definition 2.1. ( $T$ is an affine $k$-scheme.)

(1) To an object

$$
\underline{\mathcal{E}}=\left(\mathcal{E}, \mathcal{E} \otimes \mathcal{E} \rightarrow L_{T},\left\{\left.\mathcal{R}_{i} \subset \mathcal{E}\right|_{s_{i} \times T}\right\}_{i=1}^{m}\right)
$$

of $\mathcal{P} \mathcal{S U}\left(C, L ; s_{1}, \ldots, s_{m}\right)(T)$, we associate an object

$$
\underline{\mathcal{E}^{\prime}}=\left(\mathcal{E}^{\prime}, \mathcal{E}^{\prime} \otimes \mathcal{E}^{\prime} \rightarrow L\left(-s_{1}\right)_{T},\left\{\left.\mathcal{R}_{i}^{\prime} \subset \mathcal{E}^{\prime}\right|_{\left\{s_{i}\right\} \times T}\right\}_{i=1}^{m}\right)
$$


of $\mathcal{P S U}\left(C, L\left(-s_{1}\right) ; s_{1}, \ldots, s_{m}\right)(T)$ as follows. $\mathcal{E}^{\prime}:=\operatorname{Ker}\left(\left.\mathcal{E} \rightarrow \mathcal{E}\right|_{s_{1} \times T} / \mathcal{R}_{1}\right), \mathcal{R}_{1}^{\prime}:=$ $\operatorname{Ker}\left(\left.\left.\mathcal{E}^{\prime}\right|_{s_{1} \times T} \rightarrow \mathcal{R}_{1} \subset \mathcal{E}\right|_{s_{1} \times T}\right), \mathcal{R}_{i}^{\prime}:=\mathcal{R}_{i}$ for $(i \geq 2)$, and $\mathcal{E}^{\prime} \otimes \mathcal{E}^{\prime} \rightarrow L\left(-s_{1}\right)_{T}$ is the one induced by $\mathcal{E} \otimes \mathcal{E} \rightarrow L_{T}$. Then this gives rise to an isomorphism

$$
\mathcal{P S U}\left(C, L ; s_{1}, \ldots, s_{m}\right) \rightarrow \mathcal{P S U}\left(C, L\left(-s_{1}\right) ; s_{1}, \ldots, s_{m}\right)
$$

of stacks.

(2) By this isomorphism, the line bundle $\Xi_{a_{1}, \ldots, a_{m}}^{(n)}$ on $\mathcal{P S U}\left(C, L ; s_{1}, \ldots, s_{m}\right)$ corresponds to the line bundle $\Xi_{n-a_{1}, a_{2}, \ldots, a_{m}}^{(n)}$.

Proof. (1) If we apply to $\underline{\mathcal{E}^{\prime}}$ the procedure for obtaining $\underline{\mathcal{E}^{\prime}}$ from $\underline{\mathcal{E}}$, then we obtain a triple $\mathcal{E}^{\prime \prime}$ isomorphic to $\underline{\mathcal{E}} \otimes \mathcal{O}\left(-s_{1}\right)$. Hence the morphism (2.4) is an isomorphism.

(2) By the isomorphisms

$$
\left.\left.\wedge^{2} \mathcal{E}\right|_{s_{1} \times T} \simeq L_{T}\right|_{s_{1} \times T} \quad \text { and }\left.\quad \mathcal{E}^{\prime}\right|_{s_{1} \times T} / \mathcal{R}_{1}^{\prime} \simeq \mathcal{R}_{1}
$$

we have

$$
\begin{aligned}
& \left(\operatorname{det} \mathbb{R} p r_{*} \mathcal{E}\right)^{\otimes(-n)} \otimes \bigotimes_{i=1}^{m}\left(\left.\mathcal{E}\right|_{s_{i} \times T} / \mathcal{R}_{i}\right)^{\otimes a_{i}} \\
& \simeq\left(\operatorname{det} \mathbb{R} p r_{*} \mathcal{E}^{\prime}\right)^{\otimes(-n)} \otimes\left(\left.\mathcal{E}\right|_{s_{1} \times T} / \mathcal{R}_{1}\right)^{\otimes\left(a_{1}-n\right)} \otimes \bigotimes_{i=2}^{m}\left(\left.\mathcal{E}\right|_{s_{i} \times T} / \mathcal{R}_{i}\right)^{\otimes a_{i}} \\
& \simeq\left(\operatorname{det} \mathbb{R} p r_{*} \mathcal{E}^{\prime}\right)^{\otimes(-n)} \otimes\left(\left.\mathcal{E}^{\prime}\right|_{s_{1} \times T} / \mathcal{R}_{1}^{\prime}\right)^{\otimes\left(n-a_{1}\right)} \otimes \bigotimes_{i=2}^{m}\left(\left.\mathcal{E}\right|_{s_{i} \times T} / \mathcal{R}_{i}\right)^{\otimes a_{i}} \\
& \otimes_{k}\left(\left.L\right|_{s_{1}}\right)^{\otimes\left(a_{1}-n\right)} .
\end{aligned}
$$

If we fix $\left.L\right|_{s_{1}} \simeq k$, we have an isomorphism between $\Xi_{\left(a_{1}, \ldots, a_{m}\right)}^{(n)}$ on $\mathcal{P S U}\left(C, L ; s_{1}, \ldots, s_{m}\right)$ and $\Xi_{\left(n-a_{1}, a_{2}, \ldots, a_{m}\right)}^{(n)}$ on $\mathcal{P S U}\left(C, L\left(-s_{1}\right) ; s_{1}, \ldots, s_{m}\right)$. (Although this isomorphism is not canonical in the sense that it depends on the choice of the isomorphism $\left.L\right|_{s_{1}} \simeq k$, this non-canonicity does not cause any trouble in this paper.)

\section{FACTORIZATION THEOREM}

In this section we recall the factorization theorem of the space of generalized theta functions that was implicitly proved in [A. pp. 678, 679, 684].

Let $C$ be a projective nodal curve with two irreducible components $C_{1}$ and $C_{2}$. Assume that $C_{1}$ and $C_{2}$ are smooth and that they intersect at only one point $p$. Let $s$ be a closed point on $C_{1} \backslash\{p\}$. Fix a line bundle $L$ on $C$ and put $L_{i}:=\left.L\right|_{C_{i}}$. Let $g_{i}$ be the genus of $C_{i}$, and $d_{i}$ the degree of $L_{i}$.

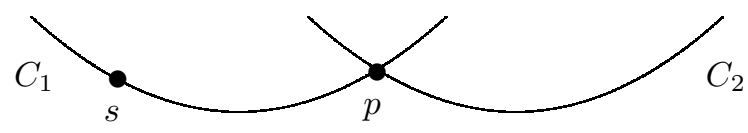

Proposition 3.1. Let $n \geq a \geq 0$ be integers. Put $\mathcal{P S U}:=\mathcal{P S U}(C, L ; s), \mathcal{P} \mathcal{S U}_{1}:=$ $\mathcal{P S U}\left(C_{1}, L_{1} ; s, p\right)$ and $\mathcal{P S U _ { 2 }}:=\mathcal{P S U}\left(C_{2}, L_{2} ; p\right)$. There exist a filtration

$$
\mathrm{H}^{0}\left(\mathcal{P S U}, \Xi_{a}^{(n)}\right)=V_{n} \supset V_{n-1} \supset \cdots \supset V_{1} \supset V_{0} \supset V_{-1}=\{0\}
$$


and an isomorphism

$$
V_{j} / V_{j-1} \simeq \mathrm{H}^{0}\left(\mathcal{P} \mathcal{S U}_{1}, \Xi_{a, j}^{(n)}\right) \otimes \mathrm{H}^{0}\left(\mathcal{P} \mathcal{S U}_{2}, \Xi_{j}^{(n)}\right)
$$

for $0 \leq j \leq n$.

The filtration and the isomorphism above are compatible with multiplication in the following sense. Let $n^{\prime} \geq a^{\prime} \geq 0$ be integers, and put $n^{\prime \prime}:=n+n^{\prime}$ and $a^{\prime \prime}:=$ $a+a^{\prime}$. Let $\left\{V_{j^{\prime}}^{\prime}\right\}_{0 \leq j^{\prime} \leq n^{\prime}}$ and $\left\{V_{j^{\prime \prime}}^{\prime \prime}\right\}_{0 \leq j^{\prime \prime} \leq n^{\prime \prime}}$ be the filtrations of $\mathrm{H}^{0}\left(\mathcal{P S U}, \Xi_{a^{\prime}}^{\left(n^{\prime}\right)}\right)$ and $\mathrm{H}^{0}\left(\mathcal{P S U}, \Xi_{a^{\prime \prime}}^{\left(n^{\prime \prime}\right)}\right)$ as above. Then we have a commutative diagram

$$
\begin{aligned}
& \mathrm{H}^{0}\left(\mathcal{P} \mathcal{S U}, \Xi_{a}^{(n)}\right) \otimes \mathrm{H}^{0}\left(\mathcal{P} \mathcal{S U}, \Xi_{a^{\prime}}^{\left(n^{\prime}\right)}\right) \quad \rightarrow \quad \mathrm{H}^{0}\left(\mathcal{P} \mathcal{S U}, \Xi_{a^{\prime \prime}}^{\left(n^{\prime \prime}\right)}\right) \\
& V_{j} \otimes V_{j^{\prime}}^{\prime} \\
& \stackrel{(*)}{\longrightarrow} \quad V_{j+j^{\prime}}^{\prime \prime}
\end{aligned}
$$

Moreover we have a commutative diagram

$$
\begin{aligned}
& V_{j} / V_{j-1} \otimes V_{j^{\prime}}^{\prime} / V_{j^{\prime}-1}^{\prime} \quad \stackrel{(* *)}{\longrightarrow} \quad V_{j+j^{\prime}}^{\prime \prime} / V_{j+j^{\prime}-1}^{\prime \prime}
\end{aligned}
$$

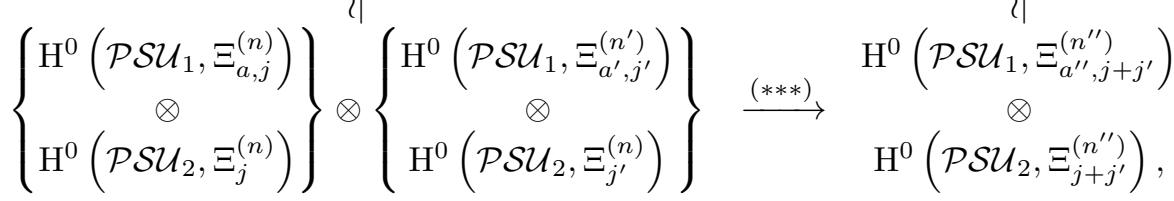

where the vertical isomorphisms are given by (3.2), (**) is the one induced by (*), and $(* * *)$ is a multiplication map.

In the later sections we use only the statement of the theorem. The reader may skip the following sketch of the proof.

Sketch of proof. Here we just recall from [A] what the filtration is, giving explicit references to $\mathrm{A}$ for technical points.

Given 2-dimensional vector spaces $W_{1}$ and $W_{2}$ together with an isomorphism $\delta: \wedge^{2} W_{1} \rightarrow \wedge^{2} W_{2}$, let us denote by $S L\left(W_{1}, W_{2}\right)$ the affine variety parametrizing isomorphisms $g: W_{1} \rightarrow W_{2}$ such that $\wedge^{2} g=\delta$. By $\overline{S L}\left(W_{1}, W_{2}\right)$ we denote the projective variety that parametrizes 2-dimensional subspaces $U \subset W_{1} \oplus W_{2}$ such that

$$
\delta \circ \wedge^{2}\left(\left.p r_{1}\right|_{U}\right)=\wedge^{2}\left(\left.p r_{2}\right|_{U}\right),
$$

where $\mathrm{pr}_{i}: W_{1} \oplus W_{2} \rightarrow W_{i}$ is the $i$-th projection. $\overline{S L}\left(W_{1}, W_{2}\right)$ is a compactification of $S L\left(W_{1}, W_{2}\right)$ which is isomorphic to a 3-dimensional quadric hypersurface, and the complement

$$
\mathbb{B}\left(W_{1}, W_{2}\right):=\overline{S L}\left(W_{1}, W_{2}\right) \backslash S L\left(W_{1}, W_{2}\right)
$$

is isomorphic to $\mathbb{P}\left(W_{1}\right) \times \mathbb{P}\left(W_{2}\right)$. (Given $\left\{M_{i} \subset W_{i}\right\}_{i=1,2}$ with $\operatorname{dim} M_{i}=1$, then $M_{1} \oplus M_{2} \subset W_{1} \oplus W_{2}$ gives a point of $\mathbb{B}\left(W_{1}, W_{2}\right)$.)

For a $(k$-valued) point

$$
\left(\left(E_{1}, E_{1} \otimes E_{1} \rightarrow L_{1},\left.R \subset E_{1}\right|_{s}\right),\left(E_{2}, E_{2} \otimes E_{2} \rightarrow L_{2}\right)\right)
$$

of $\mathcal{P S U}\left(C_{1}, L_{1} ; s\right) \times \mathcal{P} \mathcal{S U}\left(C_{2}, L_{2}\right)$, we have a natural isomorphism

$$
\left.\left.\left.\left.\wedge^{2} E_{1}\right|_{p} \simeq L_{1}\right|_{p} \simeq L_{2}\right|_{p} \simeq \wedge^{2} E_{2}\right|_{p}
$$


Hence $\overline{S L}\left(\left.E_{1}\right|_{p},\left.E_{2}\right|_{p}\right)$ makes sense here. Let $\overline{\mathcal{S L}}$ be the moduli stack that parametrizes data

$$
\left(\left(E_{1}, E_{1} \otimes E_{1} \rightarrow L_{1},\left.R \subset E_{1}\right|_{s}\right),\left(E_{2}, E_{2} \otimes E_{2} \rightarrow L_{2}\right),\left.\left.U \subset E_{1}\right|_{p} \oplus E_{2}\right|_{p}\right),
$$

where

$$
\left(\left(E_{1}, E_{1} \otimes E_{1} \rightarrow L_{1},\left.R \subset E_{1}\right|_{s}\right),\left(E_{2}, E_{2} \otimes E_{2} \rightarrow L_{2}\right)\right)
$$

is a point of $\mathcal{P S U}\left(C_{1}, L_{1} ; s\right) \times \mathcal{P S U}\left(C_{2}, L_{2}\right)$, and $\left.\left.U \subset E_{1}\right|_{p} \oplus E_{2}\right|_{p}$ is a point of $\overline{S L}\left(\left.E_{1}\right|_{p},\left.E_{2}\right|_{p}\right) . \overline{\mathcal{S L}}$ has the open substack $\mathcal{S} \mathcal{L}$ and the closed substack $\mathbb{B}$, that are defined respectively by the condition that $\left.\left.U \subset E_{1}\right|_{p} \oplus E_{2}\right|_{p}$ is in $S L\left(\left.E_{1}\right|_{p},\left.E_{2}\right|_{p}\right)$ or $\mathbb{B}\left(\left.E_{1}\right|_{p},\left.E_{2}\right|_{p}\right)$. We denote by $\zeta$ the projection $\overline{\mathcal{S} \mathcal{L}} \rightarrow \mathcal{P S U}\left(C_{1}, L_{1} ; s\right) \times \mathcal{P} \mathcal{S U}\left(C_{2}, L_{2}\right)$.

Given data (3.6), we define the torsion-free sheaf $E$ on $C$ by the short exact sequence

$$
0 \rightarrow E \rightarrow E_{1} \oplus E_{2} \rightarrow\left(\left.\left.E_{1}\right|_{p} \oplus E_{2}\right|_{p}\right) / U \rightarrow 0,
$$

where $\left(\left.\left.E_{1}\right|_{p} \oplus E_{2}\right|_{p}\right) / U$ is considered as a skyscraper sheaf at $p \in C$. We can also associate suitably a bilinear form

$$
E \otimes E \rightarrow L
$$

(see [A, Claim 3.5.3.1] for details).

We have obtained data $\left(E, E \otimes E \rightarrow L,\left.R \subset E\right|_{s}=\left.E_{1}\right|_{s}\right)$ from the data (3.6), which gives rise to a morphism $\bar{f}: \overline{\mathcal{S L}} \rightarrow \mathcal{P S \mathcal { U }}$.

Moreover we have the diagram:

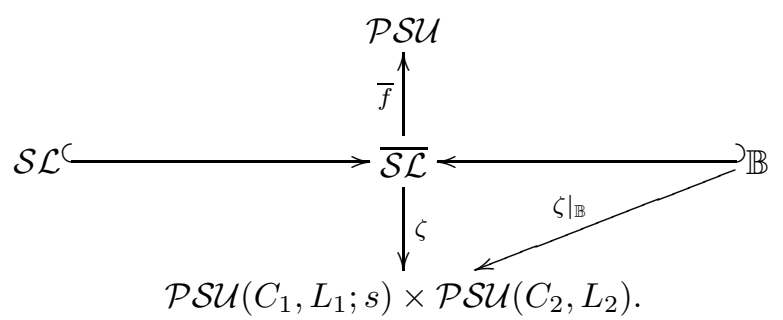

Here $\left.\zeta\right|_{\mathbb{B}}: \mathbb{B} \rightarrow \mathcal{P S U}\left(C_{1}, L_{1} ; s\right) \times \mathcal{P S U}\left(C_{2}, L_{2}\right)$ is a $\mathbb{P}^{1} \times \mathbb{P}^{1}$ bundle. In fact, $\mathbb{B}$ is nothing but $\mathcal{P} \mathcal{S U}_{1} \times \mathcal{P} \mathcal{S U}_{2}$, and $\left.\zeta\right|_{\mathbb{B}}$ is the forgetful morphism of the parabolic structure at $p$.

We have an isomorphism (cf. [A, Lemma 3.5.7])

$$
\bar{f}^{*} \Xi_{a}^{(n)} \simeq \zeta^{*}\left(\Xi_{a}^{(n)} \otimes \Xi^{(n)}\right) \otimes \mathcal{O}_{\overline{\mathcal{S L}}}(n \mathbb{B})
$$

where $\Xi_{a}^{(n)} \otimes \Xi^{(n)}$ on the right hand side is a line bundle on $\mathcal{P} \mathcal{S U}\left(C_{1}, L_{1} ; s\right) \times$ $\mathcal{P S U}\left(C_{2}, L_{2}\right)$.

We define the filter $V_{j}$ of $\mathrm{H}^{0}\left(\mathcal{P} \mathcal{S U}, \Xi_{a}^{(n)}\right)$ by

$$
\begin{aligned}
V_{j} & :=\mathrm{H}^{0}\left(\overline{\mathcal{S L}}, \zeta^{*}\left(\Xi_{a}^{(n)} \otimes \Xi^{(n)}\right) \otimes \mathcal{O}_{\overline{\mathcal{S L}}}(j \mathbb{B})\right) \\
& \subset \mathrm{H}^{0}\left(\overline{\mathcal{S L}}, \zeta^{*}\left(\Xi_{a}^{(n)} \otimes \Xi^{(n)}\right) \otimes \mathcal{O}_{\overline{\mathcal{S L}}}(n \mathbb{B})\right) \\
& \simeq \mathrm{H}^{0}\left(\overline{\mathcal{S L}}, \bar{f}^{*} \Xi_{a}^{(n)}\right) \underset{{ }_{(*)}}{\sim} \mathrm{H}^{0}\left(\mathcal{P} \mathcal{S U}, \Xi_{a}^{(n)}\right),
\end{aligned}
$$

where the fact that $(*)$ is an isomorphism is a consequence of [A, Lemma 3.5.4]. 
The restriction of the line bundle $\zeta^{*}\left(\Xi_{a}^{(n)} \otimes \Xi^{(n)}\right) \otimes \mathcal{O} \overline{\mathcal{S L}}(j \mathbb{B})$ on $\overline{\mathcal{S L}}$ to $\mathbb{B}=$ $\mathcal{P} \mathcal{S U}_{1} \times \mathcal{P} \mathcal{S U}_{2}$ is canonically isomorphic to $\Xi_{a, j}^{(n)} \otimes \Xi_{j}^{(n)}$ (cf. [A, p. 678, line 18]). We obtain the induced morphism

$$
V_{j} / V_{j-1} \rightarrow \mathrm{H}^{0}\left(\mathcal{P} \mathcal{S U}_{1}, \Xi_{a, j}^{(n)}\right) \otimes \mathrm{H}^{0}\left(\mathcal{P} \mathcal{S U}_{2}, \Xi_{j}^{(n)}\right)
$$

This is injective. For the surjectivity, see [A, p. 684, line 23].

\section{SURJeCtivity OF MULTiPLICATION MAPS}

The main theorem is proved by induction on the genus. The following proposition deals with the starting step of the induction, that is, the genus one case.

Proposition 4.1. Let $C$ be a smooth projective curve of genus one, and $L$ a line bundle on $C$ of degree $d$.

(1) Let $s$ be a closed point of $C$. Put $\mathcal{P S U}:=\mathcal{P S U}(C, L ; s)$. Then the multiplication map

$$
\mathrm{H}^{0}\left(\mathcal{P S U}, \Xi_{2}^{(2)}\right)^{\otimes j} \otimes \mathrm{H}^{0}\left(\mathcal{P S U}, \Xi_{0}^{(2)}\right)^{\otimes(l-j)} \rightarrow \mathrm{H}^{0}\left(\mathcal{P S U}, \Xi_{2 j}^{(2 l)}\right)
$$

is surjective for $l \geq j \geq 0$.

(2) Let $s_{1}$ and $s_{2}$ be distinct closed points of $C$. Put $\mathcal{P S U}:=\mathcal{P S U}\left(C, L ; s_{1}, s_{2}\right)$. If $d$ is even, then the multiplication maps

$$
\begin{aligned}
\mathrm{H}^{0}\left(\mathcal{P S U}, \Xi_{1,1}^{(1)}\right)^{\otimes 2 j} \otimes \mathrm{H}^{0}\left(\mathcal{P S U}, \Xi_{0,2}^{(2)}\right)^{\otimes k-j} \otimes \mathrm{H}^{0} & \left(\mathcal{P S U}, \Xi_{0,0}^{(1)}\right)^{\otimes 2 l-2 k} \\
& \rightarrow \mathrm{H}^{0}\left(\mathcal{P S U}, \Xi_{2 j, 2 k}^{(2 l)}\right)
\end{aligned}
$$

and

$$
\begin{aligned}
\mathrm{H}^{0}\left(\mathcal{P S U}, \Xi_{1,1}^{(1)}\right)^{\otimes 2 j} \otimes \mathrm{H}^{0}\left(\mathcal{P S U}, \Xi_{0,2}^{(2)}\right)^{\otimes k-j} \otimes \mathrm{H}^{0} & \left(\mathcal{P S U}, \Xi_{0,0}^{(1)}\right)^{\otimes 2 l-2 k+1} \\
& \rightarrow \mathrm{H}^{0}\left(\mathcal{P S U}, \Xi_{2 j, 2 k}^{(2 l+1)}\right)
\end{aligned}
$$

are surjective for $l \geq k \geq j \geq 0$.

Proof. We repeat, with minor changes, the argument in $\S 3.2$ of $[\mathrm{A}$. The point is that in the case of genus one, the coarse moduli space of parabolic bundles on a one or two-pointed curve is $\mathbb{P}^{1}$ or $\mathbb{P}^{1} \times \mathbb{P}^{1}$ so that we can carry out an explicit calculation of multiplication maps.

(1) If $d$ is even, then the surjectivity of (4.1) follows from that of (4.2) by Lemma 2.3 (2). Therefore we may assume that $d$ is odd.

Fix a stable rank 2 bundle $E_{0}$ with determinant $L$. (Note that it is unique up to isomorphism.) Put

$$
\mathcal{P S U} \supset \mathcal{P S \mathcal { U } ^ { s s }}:=\left\{\left(E,\left.R \subset E\right|_{s}\right) \mid E \simeq E_{0}\right\} .
$$

We have $\varphi: \mathcal{P S \mathcal { U } ^ { s s }} \rightarrow \mathbb{P}\left(\left.E_{0}\right|_{s}\right)$ by associating to $\left(E,\left.R \subset E\right|_{s}\right)$ the 1-dimensional subspace $\left.\left.R \subset E\right|_{s} \simeq E_{0}\right|_{s}$. It was proved in the proof of Proposition 3.2.2 of [A] that $\varphi$ is a certain GIT quotient; hence for any line bundle $M$ on $\mathbb{P}\left(\left.E_{0}\right|_{s}\right)$ we have an isomorphism

$$
\varphi^{*}: \mathrm{H}^{0}\left(\mathbb{P}\left(\left.E_{0}\right|_{s}\right), M\right) \stackrel{\sim}{\rightarrow} \mathrm{H}^{0}\left(\mathcal{P} \mathcal{S} \mathcal{U}^{s s}, \varphi^{*} M\right)
$$


By construction, we have isomorphisms $\varphi^{*} \mathcal{O}(1) \simeq \Xi_{1}^{(1)}$ and $\varphi^{*} \mathcal{O} \simeq \Xi_{0}^{(1)}$. By the Verlinde formula (cf. [B3]), we have $\operatorname{dim} \mathrm{H}^{0}\left(\mathcal{P S U}, \Xi_{a}^{(n)}\right)=a+1$ for $n \geq a \geq 0$. Hence the inclusion $\mathrm{H}^{0}\left(\mathcal{P} \mathcal{S U}, \Xi_{a}^{(n)}\right) \hookrightarrow \mathrm{H}^{0}\left(\mathcal{P} \mathcal{S} \mathcal{U}^{s s}, \Xi_{a}^{(n)}\right)$ is in fact an isomorphism 11 We have isomorphisms

$$
\mathrm{H}^{0}\left(\mathbb{P}\left(\left.E_{0}\right|_{s}\right), \mathcal{O}(a)\right) \stackrel{\sim}{\rightarrow} \mathrm{H}^{0}\left(\mathcal{P} \mathcal{S} \mathcal{U}^{s s}, \Xi_{a}^{(n)}\right) \leftarrow \mathrm{H}^{0}\left(\mathcal{P} \mathcal{S U}, \Xi_{a}^{(n)}\right) .
$$

Therefore the surjectivity of (4.1) follows from that of

$$
\mathrm{H}^{0}\left(\mathbb{P}^{1}, \mathcal{O}(2)\right)^{\otimes j} \otimes \mathrm{H}^{0}\left(\mathbb{P}^{1}, \mathcal{O}\right)^{\otimes l-j} \rightarrow \mathrm{H}^{0}\left(\mathbb{P}^{1}, \mathcal{O}(2 j)\right) .
$$

(2) By performing an elementary transformation at $s_{2}$, we know by Lemma 2.4 that it suffices to prove that for $d$ odd, the multiplication maps

$$
\begin{aligned}
& \mathrm{H}^{0}\left(\mathcal{P S U}, \Xi_{1,0}^{(1)}\right)^{\otimes 2 j} \otimes \mathrm{H}^{0}\left(\mathcal{P} \mathcal{S U}, \Xi_{0,0}^{(2)}\right)^{\otimes k-j} \otimes \mathrm{H}^{0}\left(\mathcal{P} \mathcal{S U}, \Xi_{0,1}^{(1)}\right)^{\otimes 2 l-2 k} \\
& \rightarrow \mathrm{H}^{0}\left(\mathcal{P S U}, \Xi_{2 j, 2 l-2 k}^{(2 l)}\right)
\end{aligned}
$$

and

$$
\begin{aligned}
& \mathrm{H}^{0}\left(\mathcal{P S U}, \Xi_{1,0}^{(1)}\right)^{\otimes 2 j} \otimes \mathrm{H}^{0}\left(\mathcal{P S U}, \Xi_{0,0}^{(2)}\right)^{\otimes k-j} \otimes \mathrm{H}^{0}\left(\mathcal{P S U}, \Xi_{0,1}^{(1)}\right)^{\otimes 2 l-2 k+1} \\
& \rightarrow \mathrm{H}^{0}\left(\mathcal{P} \mathcal{S} \mathcal{U}, \Xi_{2 j, 2 l-2 k+1}^{(2 l+1)}\right)
\end{aligned}
$$

are surjective. Let $E_{0}$ be a rank 2 stable vector bundle with determinant $L$. Put $\mathcal{P S U} \supset \mathcal{P S U}^{s s}:=\left\{\left(E,\left.R_{1} \subset E\right|_{s_{1}},\left.R_{2} \subset E\right|_{s_{2}}\right) \mid E \simeq E_{0}\right\}$. We have a morphism $\varphi: \mathcal{P S U} \mathcal{S}^{s s} \rightarrow \mathbb{P}\left(\left.E_{0}\right|_{s_{1}}\right) \times \mathbb{P}\left(\left.E_{0}\right|_{s_{2}}\right)$ by associating

$$
\left(\left.\left.R_{1} \subset E\right|_{s_{1}} \simeq E_{0}\right|_{s_{1}},\left.\left.R_{2} \subset E\right|_{s_{2}} \simeq E_{0}\right|_{s_{2}}\right)
$$

to $\left(E,\left.R_{1} \subset E\right|_{s_{1}},\left.R_{2} \subset E\right|_{s_{2}}\right)$. Since $\varphi$ is a certain GIT quotient, we have an isomorphism

$$
\varphi^{*}: \mathrm{H}^{0}\left(\mathbb{P}\left(\left.E_{0}\right|_{s_{1}}\right) \times \mathbb{P}\left(\left.E_{0}\right|_{s_{2}}\right), M\right) \stackrel{\sim}{\rightarrow} \mathrm{H}^{0}\left(\mathcal{P S \mathcal { U } ^ { s s }}, \varphi^{*} M\right)
$$

for any line bundle $M$ on $\mathbb{P}\left(\left.E_{0}\right|_{s_{1}}\right) \times \mathbb{P}\left(\left.E_{0}\right|_{s_{2}}\right)$. If $n+a_{1}+a_{2}$ is even, the line bundle $\left.\Xi_{a_{1}, a_{2}}^{(n)}\right|_{\mathcal{P S} \mathcal{U}^{s s}}$ on $\mathcal{P} \mathcal{S U}^{s s}$ descends to a line bundle $\mathcal{O}\left(a_{1}\right) \otimes \mathcal{O}\left(a_{2}\right)$ on $\mathbb{P}\left(\left.E_{0}\right|_{s_{1}}\right) \times$ $\mathbb{P}\left(\left.E_{0}\right|_{s_{2}}\right)$. Moreover if $a_{1}+a_{2} \leq n$, then by the Verlinde formula we have

$$
\operatorname{dim} H^{0}\left(\mathcal{P S U}, \Xi_{a_{1}, a_{2}}^{(n)}\right)=\left(a_{1}+1\right)\left(a_{2}+1\right) .
$$

Hence we have

$$
\begin{aligned}
\mathrm{H}^{0}\left(\mathbb{P}\left(\left.E_{0}\right|_{s_{1}}\right) \times \mathbb{P}\left(\left.E_{0}\right|_{s_{2}}\right), \mathcal{O}\left(a_{1}\right) \otimes \mathcal{O}\left(a_{2}\right)\right) & \stackrel{\sim}{\rightarrow} \mathrm{H}^{0}\left(\mathcal{P S \mathcal { U } ^ { s s }}, \Xi_{a_{1}, a_{2}}^{(n)} \mid{ }_{\mathcal{P S}} \mathcal{U}^{s s}\right) \\
& \sim \mathrm{H}^{0}\left(\mathcal{P S U}, \Xi_{a_{1}, a_{2}}^{(n)}\right) .
\end{aligned}
$$

Therefore the surjectivity of (4.5) and (4.6) follows from that of

$$
\begin{aligned}
& \mathrm{H}^{0}\left(\mathbb{P}^{1} \times \mathbb{P}^{1}, \mathcal{O}(1) \otimes \mathcal{O}\right)^{\otimes 2 j} \otimes \mathrm{H}^{0}\left(\mathbb{P}^{1} \times \mathbb{P}^{1}, \mathcal{O} \otimes \mathcal{O}\right)^{\otimes k-j} \\
& \otimes \mathrm{H}^{0}\left(\mathbb{P}^{1} \times \mathbb{P}^{1}, \mathcal{O} \otimes \mathcal{O}(1)\right)^{\otimes 2 l-2 k} \rightarrow \mathrm{H}^{0}\left(\mathbb{P}^{1} \times \mathbb{P}^{1}, \mathcal{O}(2 j) \otimes \mathcal{O}(2 l-2 k)\right)
\end{aligned}
$$

\footnotetext{
${ }^{1}$ By a general fact on GIT quotients [N-Rd Lemma 4.15], we can infer that it is an isomorphism without appealing to the Verlinde formula.
} 
and

$$
\begin{aligned}
& \mathrm{H}^{0}\left(\mathbb{P}^{1} \times \mathbb{P}^{1}, \mathcal{O}(1) \otimes \mathcal{O}\right)^{\otimes 2 j} \otimes \mathrm{H}^{0}\left(\mathbb{P}^{1} \times \mathbb{P}^{1}, \mathcal{O} \otimes \mathcal{O}\right)^{\otimes k-j} \\
& \otimes \mathrm{H}^{0}\left(\mathbb{P}^{1} \times \mathbb{P}^{1}, \mathcal{O} \otimes \mathcal{O}(1)\right)^{\otimes 2 l-2 k+1} \rightarrow \mathrm{H}^{0}\left(\mathbb{P}^{1} \times \mathbb{P}^{1}, \mathcal{O}(2 j) \otimes \mathcal{O}(2 l-2 k+1)\right) .
\end{aligned}
$$

Now we come to the main theorem of this paper.

Theorem 4.2. Fix a positive integer $l$. Let $(C, s)$ be a generic smooth projective 1-pointed curve of genus $g \geq 1$. Let $L$ be a line bundle on $C$ of degree $d$. Put $\mathcal{P S U}:=\mathcal{P S U}(C, L ; s)$. Then the multiplication map

$$
\mathrm{H}^{0}\left(\mathcal{P} \mathcal{S U}, \Xi_{2}^{(2)}\right)^{\otimes j} \otimes \mathrm{H}^{0}\left(\mathcal{P} \mathcal{S U}, \Xi_{0}^{(2)}\right)^{\otimes l-j} \rightarrow \mathrm{H}^{0}\left(\mathcal{P} \mathcal{S U}, \Xi_{2 j}^{(2 l)}\right)
$$

is surjective for $l \geq j \geq 0$.

Moreover, if $d$ is even, then

$$
\begin{aligned}
\mathrm{H}^{0}\left(\mathcal{P} \mathcal{S U}, \Xi_{0}^{(1)}\right) \otimes \mathrm{H}^{0}\left(\mathcal{P} \mathcal{S U}, \Xi_{2}^{(2)}\right)^{\otimes j} & \otimes \mathrm{H}^{0}\left(\mathcal{P S U}, \Xi_{0}^{(2)}\right)^{\otimes l-j} \\
& \rightarrow \mathrm{H}^{0}\left(\mathcal{P S U}, \Xi_{2 j}^{(2 l+1)}\right)
\end{aligned}
$$

is surjective for $l \geq j \geq 0$.

Proof. We proceed by induction on $g \geq 1$. If $g=1$, then the surjectivity of (4.10) and (4.11) follows from Proposition 4.1 .

Now let $g \geq 2$, and assume that the theorem is true for $g-1$. Let $C_{1} \cup C_{2}$ be a nodal curve of genus $g$ such that $C_{1}$ is a smooth projective curve of genus one, $C_{2}$ is a smooth projective curve of genus $g-1$, and that $C_{1}$ and $C_{2}$ intersect at only one point $p$. Let $s$ be a closed point of $C_{1} \backslash\{p\}$, and $L$ a line bundle on $C_{1} \cup C_{2}$ such that $\left.\operatorname{deg} L\right|_{C_{1}}=0$ and $\left.\operatorname{deg} L\right|_{C_{2}}=d$. Put $\mathcal{P S U}:=\mathcal{P} \mathcal{S U}\left(C_{1} \cup C_{2}, L ; s\right)$, $\mathcal{P S \mathcal { U } _ { 1 }}:=\mathcal{P} \mathcal{S U}\left(C_{1},\left.L\right|_{C_{1}} ; s, p\right)$ and $\mathcal{P} \mathcal{S U}_{2}:=\mathcal{P} \mathcal{S U}\left(C_{2},\left.L\right|_{C_{2}} ; p\right)$. Supposing that the maps (4.10) and (4.11) are surjective for $\mathcal{P} \mathcal{S U}_{2}$, let us prove that (4.10) and (4.11) are surjective for $\mathcal{P S U}$. For $n \geq a \geq 0$, let

$$
\mathrm{H}^{0}\left(\mathcal{P S U}, \Xi_{a}^{(n)}\right)=V_{a, n}^{(n)} \supset V_{a, n-1}^{(n)} \supset \cdots \supset V_{a, 1}^{(n)} \supset V_{a, 0}^{(n)} \supset V_{a,-1}^{(n)}=\{0\}
$$

be the filtration given by Proposition 3.1. By the compatibility of this filtration with the multiplication stated in Proposition 3.1. the surjectivity of (4.10) and (4.11) follows from that of

$$
\bigoplus_{b_{1}+\ldots b_{j}+c_{1}+\cdots+c_{l-j}=2 r} \bigotimes_{\alpha=1}^{j} V_{2, b_{\alpha}}^{(2)} / V_{2, b_{\alpha}-1}^{(2)} \otimes \bigotimes_{\beta=1}^{l-j} V_{0, c_{\beta}}^{(2)} / V_{0, c_{\beta}-1}^{(2)} \rightarrow V_{2 j, 2 r}^{(2 l)} / V_{2 j, 2 r-1}^{(2 l)}
$$

and

$$
\begin{aligned}
\bigoplus_{b_{1}+\ldots b_{j}+c_{1}+\cdots+c_{l-j}=2 r} V_{0,0}^{(1)} / V_{0,-1}^{(1)} \otimes \bigotimes_{\alpha=1}^{j} V_{2, b_{\alpha}}^{(2)} / V_{2, b_{\alpha}-1}^{(2)} & \otimes \bigotimes_{\beta=1}^{l-j} V_{0, c_{\beta}}^{(2)} / V_{0, c_{\beta}-1}^{(2)} \\
& \rightarrow V_{2 j, 2 r}^{(2 l+1)} / V_{2 j, 2 r-1}^{(2 l+1)},
\end{aligned}
$$


where $b_{\alpha}, c_{\beta}=0$ or 2 . For this, it suffices to prove the surjectivity of

$$
\begin{aligned}
\left(V_{2,2}^{(2)} / V_{2,1}^{(2)}\right)^{\otimes r} \otimes\left(V_{2,0}^{(2)} / V_{2,-1}^{(2)}\right)^{\otimes j-r} \otimes & \left(V_{0,0}^{(2)} / V_{0,-1}^{(2)}\right)^{\otimes l-j} \\
& \rightarrow V_{2 j, 2 r}^{(2 l)} / V_{2 j, 2 r-1}^{(2 l)}
\end{aligned}
$$

and

$$
\begin{aligned}
V_{0,0}^{(1)} / V_{0,-1}^{(1)} \otimes\left(V_{2,2}^{(2)} / V_{2,1}^{(2)}\right)^{\otimes r} \otimes\left(V_{2,0}^{(2)} / V_{2,-1}^{(2)}\right)^{\otimes j-r} \\
\otimes\left(V_{0,0}^{(2)} / V_{0,-1}^{(2)}\right)^{\otimes l-j} \rightarrow V_{2 j, 2 r}^{(2 l+1)} / V_{2 j, 2 r-1}^{(2 l+1)}
\end{aligned}
$$

if $j \geq r$, and it suffices to prove the surjectivity of

$$
\begin{aligned}
\left(V_{2,2}^{(2)} / V_{2,1}^{(2)}\right)^{\otimes j} \otimes\left(V_{0,2}^{(2)} / V_{0,1}^{(2)}\right)^{\otimes r-j} \otimes & \left(V_{0,0}^{(2)} / V_{0,-1}^{(2)}\right)^{\otimes l-r} \\
& \rightarrow V_{2 j, 2 r}^{(2 l)} / V_{2 j, 2 r-1}^{(2 l)}
\end{aligned}
$$

and

$$
\begin{aligned}
V_{0,0}^{(1)} / V_{0,-1}^{(1)} \otimes\left(V_{2,2}^{(2)} / V_{2,1}^{(2)}\right)^{\otimes j} \otimes\left(V_{0,2}^{(2)} / V_{0,1}^{(2)}\right)^{\otimes r-j} \\
\otimes\left(V_{0,0}^{(2)} / V_{0,-1}^{(2)}\right)^{\otimes l-r} \rightarrow V_{2 j, 2 r}^{(2 l+1)} / V_{2 j, 2 r-1}^{(2 l+1)}
\end{aligned}
$$

if $r \geq j$. By the commutative diagram (3.4), the surjectivity of these maps is equivalent to that of

$$
\begin{aligned}
& \left\{\begin{array}{c}
\mathrm{H}^{0}\left(\mathcal{P} \mathcal{S U}_{1}, \Xi_{2,2}^{(2)}\right) \\
\otimes \\
\mathrm{H}^{0}\left(\mathcal{P} \mathcal{S U}_{2}, \Xi_{2}^{(2)}\right)
\end{array}\right\} \otimes\left\{\begin{array}{c}
\mathrm{H}^{0}\left(\mathcal{P} \mathcal{S U}_{1}, \Xi_{2,0}^{(2)}\right) \\
\otimes \\
\mathrm{H}^{0}\left(\mathcal{P} \mathcal{S U}_{2}, \Xi_{0}^{(2)}\right)
\end{array}\right\} \otimes \otimes\left\{\begin{array}{c}
\mathrm{H}^{0}\left(\mathcal{P} \mathcal{S U}_{1}, \Xi_{0,0}^{(2)}\right) \\
\otimes \\
\mathrm{H}^{0}\left(\mathcal{P} \mathcal{S U}_{2}, \Xi_{0}^{(2)}\right)
\end{array}\right\}^{\otimes j-r} \\
& \mathrm{H}^{0}\left(\mathcal{P} \mathcal{S U}_{1}, \Xi_{2 j, 2 r}^{(2 l)}\right) \\
& \rightarrow \quad \otimes \\
& \mathrm{H}^{0}\left(\mathcal{P S U}_{2}, \Xi_{2 r}^{(2 l)}\right),
\end{aligned}
$$

$$
\begin{aligned}
& \left.\left\{\begin{array}{c}
\mathrm{H}^{0}\left(\mathcal{P} \mathcal{S U}_{1}, \Xi_{0,0}^{(1)}\right) \\
\otimes \\
\mathrm{H}^{0}\left(\mathcal{P} \mathcal{S U}_{2}, \Xi_{0}^{(1)}\right)
\end{array}\right\} \otimes\left\{\begin{array}{c}
\mathrm{H}^{0}\left(\mathcal{P} \mathcal{S U}_{1}, \Xi_{2,2}^{(2)}\right) \\
\otimes \\
\mathrm{H}^{0}\left(\mathcal{P} \mathcal{S U}_{2}, \Xi_{2}^{(2)}\right)
\end{array}\right\}^{\otimes r} \otimes\left\{\begin{array}{c}
\mathrm{H}^{0}\left(\mathcal{P} \mathcal{S U}_{1}, \Xi_{2,0}^{(2)}\right) \\
\otimes \\
\mathrm{H}^{0}\left(\mathcal{P} \mathcal{S U}_{2}, \Xi_{0}^{(2)}\right)
\end{array}\right\}\right\}^{\otimes j-r} \\
& \otimes\left\{\begin{array}{c}
\mathrm{H}^{0}\left(\mathcal{P} \mathcal{S U}_{1}, \Xi_{0,0}^{(2)}\right) \\
\otimes \\
\mathrm{H}^{0}\left(\mathcal{P} \mathcal{S U}_{2}, \Xi_{0}^{(2)}\right)
\end{array}\right\} \quad \begin{array}{r}
\mathrm{H}^{0}\left(\mathcal{P S U}_{1}, \Xi_{2 j, 2 r}^{(2 l+1)}\right) \\
\mathrm{H}^{0}\left(\mathcal{P} \mathcal{S U}_{2}, \Xi_{2 r}^{(2 l+1)}\right)
\end{array}
\end{aligned}
$$


if $j \geq r$, and

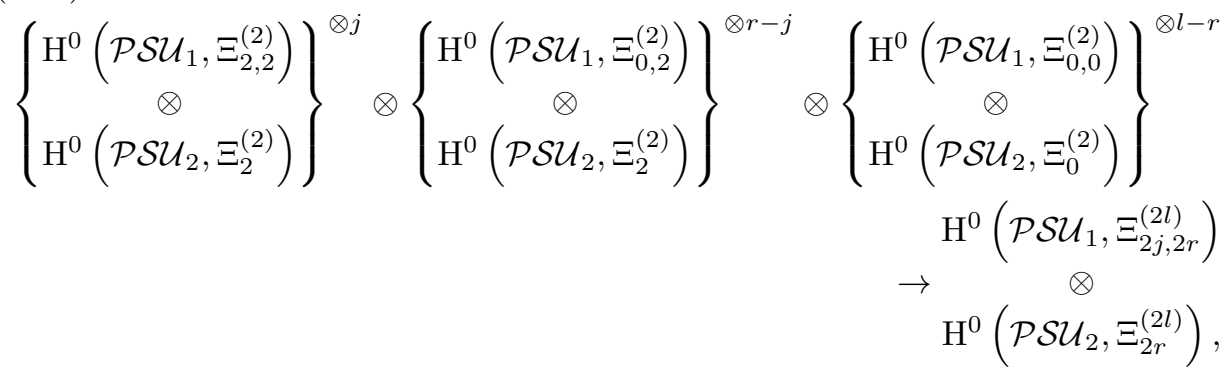

$$
\begin{aligned}
& \left\{\begin{array}{c}
\mathrm{H}^{0}\left(\mathcal{P} \mathcal{S U}_{1}, \Xi_{0,0}^{(1)}\right) \\
\otimes \\
\mathrm{H}^{0}\left(\mathcal{P} \mathcal{S U}_{2}, \Xi_{0}^{(1)}\right)
\end{array}\right\} \otimes\left\{\begin{array}{c}
\mathrm{H}^{0}\left(\mathcal{P} \mathcal{S U}_{1}, \Xi_{2,2}^{(2)}\right) \\
\otimes \\
\mathrm{H}^{0}\left(\mathcal{P} \mathcal{S U}_{2}, \Xi_{2}^{(2)}\right)
\end{array}\right\} \otimes j \quad\left\{\begin{array}{c}
\mathrm{H}^{0}\left(\mathcal{P} \mathcal{S U}_{1}, \Xi_{0,2}^{(2)}\right) \\
\otimes \\
\mathrm{H}^{0}\left(\mathcal{P} \mathcal{S U}_{2}, \Xi_{2}^{(2)}\right)
\end{array}\right\}^{\otimes r-j}
\end{aligned}
$$

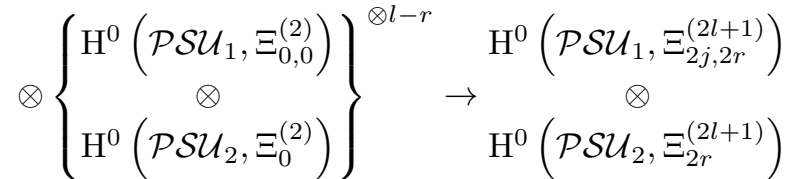

if $r \geq j$.

By the induction hypothesis and Proposition 4.1, these maps are surjective.

By smoothing $C_{1} \cup C_{2}$, we can infer that the theorem is true for $g$ as follows. Let $\pi: \mathcal{C} \rightarrow S=$ Spec $k[[t]]$ be a projective flat morphism, and let $\mathcal{L}$ be a line bundle on $\mathcal{C}$. Let $o \in S$ be the closed point, and $\eta$ the generic point. Let $\tilde{s}: S \rightarrow \mathcal{C}$ be a section of $\pi$. Assume that $\mathcal{C} \times{ }_{S} \eta \rightarrow \eta$ is a smooth projective curve, and that $\mathcal{C} \times{ }_{S} O \rightarrow o$ is $C_{1} \cup C_{2}$ with $s=\tilde{s}(o)$ and $\left.\mathcal{L}\right|_{\mathcal{C}_{\times_{S} o}} \simeq L$ as above. We can extend straightforwardly the definition of the moduli stack of parabolic 2-bundles with fixed determinant and the line bundle $\Xi_{a}^{(n)}$ in Definition 2.1 to this relative setting. Thus we obtain the moduli stack $\widetilde{\mathcal{P S U}}:=\mathcal{P S \mathcal { U }}(\mathcal{C} / S, \mathcal{L} ; \tilde{s})$ over $S$ and a line bundle $\widetilde{\Xi_{a}^{(n)}}$ on it. (Here we add a tilde to indicate that it is a line bundle on the relative moduli $\mathcal{P} \mathcal{S U}(\mathcal{C} / S, \mathcal{L} ; \tilde{s})$.) In [A, p. 685], it is proved that $\mathrm{H}^{0}\left(\widetilde{\mathcal{P S U}}, \widetilde{\Xi_{a}^{(n)}}\right)$ is a finite free $k[[t]]$-module, and that the morphisms

$$
\mathrm{H}^{0}\left(\widetilde{\mathcal{P S U}}, \widetilde{\Xi_{a}^{(n)}}\right) \otimes k[[t]] /(t) \rightarrow \mathrm{H}^{0}\left(\widetilde{\mathcal{P S U}} \times_{S} o,\left.\widetilde{\Xi_{a}^{(n)}}\right|_{\widetilde{\mathcal{P U}} \times{ }_{S} o}\right)
$$

and

$$
\mathrm{H}^{0}\left(\widetilde{\mathcal{P S U}}, \widetilde{\Xi_{a}^{(n)}}\right) \otimes k((t)) \rightarrow \mathrm{H}^{0}\left(\widetilde{\mathcal{P S U}} \times_{S} \eta,\left.\widetilde{\Xi_{a}^{(n)}}\right|_{\widetilde{\mathcal{S U}} \times{ }_{S} \eta}\right)
$$

are isomorphisms. (See the remark below about this point.)

Therefore the surjectivity of (4.10) and (4.11) for $\mathcal{P S U}\left(C_{1} \cup C_{2}, L ; s\right)=\widetilde{\mathcal{P S U}} \times{ }_{S} O$ implies that for $\widetilde{\mathcal{P S U}} \times{ }_{S} \eta$.

Remark 4.3. The isomorphisms (4.23) and (4.24) ensure that under degeneration the dimension of the space of generalized theta functions does not jump. This fact 
is one of the crucial points for proving the Verlinde formula by the degeneration method. For example, in the case of the moduli of rank 2 vector bundles with non-fixed determinant, Narasimhan and Ramadas [N-Rd], $\mathrm{Rd}$ derived the fact by proving the vanishing of the first cohomology of the generalized theta divisor. However in [A, the author proved the isomorphisms (4.23) and (4.24) using the Verlinde formula. Since the Verlinde formula is now established, this is logically no problem (although it might give the impression of cheating).

Corollary 4.4. Let $C$ be a generic smooth projective curve of genus $g \geq 2$. Then the ample generator $\mathcal{L}$ of $\operatorname{Pic} S U_{C}(2)$ gives a projectively normal embedding $\varphi_{\mathcal{L}}$ : $S U_{C}(2) \rightarrow \mathbb{P}\left(\mathrm{H}^{0}\left(S U_{C}(2), \mathcal{L}\right)\right)$.

Proof. It suffices to prove that the multiplication map

$$
S^{n} \mathrm{H}^{0}\left(S U_{C}(2), \mathcal{L}\right) \rightarrow \mathrm{H}^{0}\left(S U_{C}(2), \mathcal{L}^{\otimes n}\right)
$$

is surjective for $n \geq 1$. First note that the family of smooth projective curves of genus $g$ is bounded, so there exists an $n_{0}$ such that for $\forall n \geq n_{0}$ the map (4.25) is surjective for all smooth projective curves of genus $g$. So we have only to prove that for a fixed $n$, the map (4.25) is surjective for a generic $C$. By B-L, Proposition 8.3 and Proposition 8.4], we have a natural isomorphism

$$
\mathrm{H}^{0}\left(S U_{C}(2), \mathcal{L}^{\otimes n}\right) \simeq \mathrm{H}^{0}\left(\mathcal{P S U}\left(C, \mathcal{O}_{C}\right), \Xi^{(n)}\right) .
$$

This isomorphism and Theorem 4.2 imply that for a fixed $l$, the multiplication maps

$$
S^{l} \mathrm{H}^{0}\left(S U_{C}(2), \mathcal{L}^{2}\right) \rightarrow \mathrm{H}^{0}\left(S U_{C}(2), \mathcal{L}^{\otimes 2 l}\right)
$$

and

$$
\mathrm{H}^{0}\left(S U_{C}(2), \mathcal{L}\right) \otimes S^{l} \mathrm{H}^{0}\left(S U_{C}(2), \mathcal{L}^{2}\right) \rightarrow \mathrm{H}^{0}\left(S U_{C}(2), \mathcal{L}^{\otimes 2 l+1}\right)
$$

are surjective for a generic $C$. Since

$$
S^{2} \mathrm{H}^{0}\left(S U_{C}(2), \mathcal{L}\right) \rightarrow \mathrm{H}^{0}\left(S U_{C}(2), \mathcal{L}^{\otimes 2}\right)
$$

is surjective for a generic $C$ by [B2], we know that for a fixed $n$, the map (4.25) is surjective.

\section{ACKNOWLEDGEMEnTS}

Part of this work was done during the author's visit at Nice University in October and November 2006, financially supported by the Japanese Ministry of Education, Culture, Sport, Science and Technology. He would like to express his deep gratitude to the staff of Nice University, especially Professor Beauville, for a wonderful research environment.

\section{REFERENCES}

[A] T. Abe: On $S L(2)-G L(n)$ strange duality, J. Math. Kyoto Univ., Vol. 46(3), 657-692, (2006). MR2311364 (2008e:14012)

[B1] A. Beauville: Fibrés de rang 2 sur une courbe, fibré déterminant et fonctions thêta, Bull. Soc. Math. France 116 (1988), no. 4, 431-448. MR.1005388 (91b:14038)

[B2] A. Beauville: Fibrés de rang deux sur une courbe, fibré déterminant et fonctions thêta II, Bull. Soc. Math. France 119 (1991), no. 3, 259-291. MR.1125667 (92m:14041)

[B3] A. Beauville: Conformal blocks, fusion rules and the Verlinde formula, Proceedings of the Hirzebruch 65 Conference on Algebraic Geometry (Ramat Gan, 1993), 75-96, Israel Math. Conf. Proc., 9, Bar-Ilan Univ., Ramat Gan, 1996. MR1360497 (97f:17025) 
[B-L] A. Beauville, Y. Laszlo: Conformal blocks and generalized theta functions, Comm. Math. Phys. 164 (1994), no. 2, 385-419. MR.1289330 (95k:14011)

[Bel] P. Belkale: The Strange Duality Conjecture for Generic Curves, math. AG/0602018.

[Bho1] U N. Bhosle: Tensor fields and singular principal bundles, Int. Math. Res. Not. 2004, no. 57, 3057-3077. MR2098029 (2006f:14048)

[Bho2] U N. Bhosle: Vector bundles with a fixed determinant on an irreducible nodal curve, Proc. Indian Acad. Sci. Math. Sci. 115 (2005), no. 4, 445-451. MR2184204 (2007e:14054)

[B-V] S. Brivio, A. Verra: The theta divisor of $\mathrm{SU}_{C}(2,2 d)^{s}$ is very ample if $C$ is not hyperelliptic, Duke Math. J. 82 (1996), no. 3, 503-552. MR.1387683 (97e:14017)

[D-N] J. M. Drezet, M. S. Narasimhan: Groupe de Picard des variétés de modules de fibrés semi-stables sur les courbes algébriques, Invent. Math. 97 (1989), no. 1, 53-94. MR999313 (90d:14008)

[F] G. Faltings: Moduli-stacks for bundles on semistable curves, Math. Ann. 304, 489-515 (1996). MR1375622 (97d:14016)

[L] Y. Laszlo: A propos de l'espace des modules de fibrés de rang 2 sur une courbe, Math. Ann. 299 (1994), no. 4, 597-608. MR.1286886 (95f:14021)

[M-O] A. Marian, D. Oprea: The level-rank duality for non-abelian theta functions, Invent. Math. 168 (2007), no. 2, 225-247. MR2289865 (2007k:14070)

[N-Rd] M. S. Narasimhan, T. R. Ramadas: Factorisation of generalised theta functions. I, Invent. Math. 114 (1993), no. 3, 565-623. MR.1244913(94i:14017)

[N-R] M. S. Narasimhan, S. Ramanan: Moduli of vector bundles on a compact Riemann surface, Ann. of Math. (2) 891969 14-51. MR0242185 (39:3518)

[Rd] T. R. Ramadas: Factorisation of generalised theta functions. II. The Verlinde formula, Topology 35 (1996), no. 3, 641-654. MR1396770 (97j:14014)

[R] M. Raynaud: Sections des fibrés vectoriels sur une courbe, Bull. Soc. Math. France 110 (1982), no. 1, 103-125. MR662131 (84a:14009)

[vG-I] B. van Geemen, E. Izadi: The tangent space to the moduli space of vector bundles on a curve and the singular locus of the theta divisor of the Jacobian, J. Algebraic Geom. 10 (2001), no. 1, 133-177. MR.1795553 (2002e:14058)

[vG-P] B. van Geemen, E. Previato: Prym varieties and the Verlinde formula, Math. Ann. 294 (1992), no. 4, 741-754. MR.1190454 (93j:14037)

[Sch1] A. H. W. Schmitt: Singular principal G-bundles on nodal curves, J. Eur. Math. Soc. (JEMS) 7 (2005), no. 2, 215-251. MR2127994 (2005k:14070)

[Sch2] A. H. W. Schmitt: Moduli spaces for semistable honest singular principal bundles on a nodal curve which are compatible with degeneration. A remark on U. N. Bhosle's paper: "Tensor fields and singular principal bundles", Int. Math. Res. Not. 2005, no. 23, 14271437. MR2152237 (2007e:14019)

[S1] X. Sun: Degeneration of moduli spaces and generalized theta functions, J. Algebraic Geom. 9 (2000), no. 3, 459-527. MR.1752012 (2001h:14040)

[S2] X. Sun: Factorization of generalized theta functions in the reducible case, Ark. Mat. 41 (2003), no. 1, 165-202. MR1971947 (2004c:14065)

Research Institute for Mathematical Sciences, Kyoto University, Kitashirakawa, SAKYO-KU, KYOTO, 606-8502, JAPAN

E-mail address: abeken@kurims.kyoto-u.ac.jp

Current address: Graduate School of Science and Technology, Kumamoto University, 2-39-1

Kurokami, Kumamoto 860-8555, Japan

E-mail address: abeken@sci.kumamoto-u.ac.jp 